\title{
Extracting Physicochemical Features to Predict Protein Secondary Structure
}

\author{
Yin-Fu Huang and Shu-Ying Chen \\ Department of Computer Science and Information Engineering, National Yunlin University of Science and Technology, \\ 123 University Road, Section 3, Touliu, Yunlin 640, Taiwan
}

Correspondence should be addressed to Yin-Fu Huang; huangyf@yuntech.edu.tw

Received 29 January 2013; Accepted 23 April 2013

Academic Editors: S. Jahandideh and J. Ni

Copyright (C) 2013 Y.-F. Huang and S.-Y. Chen. This is an open access article distributed under the Creative Commons Attribution License, which permits unrestricted use, distribution, and reproduction in any medium, provided the original work is properly cited.

\begin{abstract}
We propose a protein secondary structure prediction method based on position-specific scoring matrix (PSSM) profiles and four physicochemical features including conformation parameters, net charges, hydrophobic, and side chain mass. First, the SVM with the optimal window size and the optimal parameters of the kernel function is found. Then, we train the SVM using the PSSM profiles generated from PSI-BLAST and the physicochemical features extracted from the CB513 data set. Finally, we use the filter to refine the predicted results from the trained SVM. For all the performance measures of our method, $Q_{3}$ reaches 79.52, SOV94 reaches 86.10, and SOV99 reaches 74.60; all the measures are higher than those of the SVMpsi method and the SVMfreq method. This validates that considering these physicochemical features in predicting protein secondary structure would exhibit better performances.
\end{abstract}

\section{Introduction}

Many issues on molecular biology have been addressed in the past decades, including genetics, structural biology, and drug design. A protein primary sequence is composed of amino acids; as we know, totally 20 different kinds of amino acids can be found in protein sequences. In this paper, we would investigate protein secondary structures based on protein sequences.

The secondary structure of a protein sequence comes from different folding of amino acids, due to the differences of their side chain sizes, shapes, reactivity, and the ability to form hydrogen bonds. Furthermore, owing to the differences of the side chain sizes, the number of electric charges, coupled with the affinity for water, the tertiary structures of protein sequences are not all the same. Thus, the exploration of molecular structures on protein sequences is divided into secondary, tertiary, and even quaternary structures. Given a protein primary sequence, its corresponding secondary structure can be revealed as follows:

Primary sequence:

MFKVYGYDSNIHKCVYCDNAKRLLTVKKQPFEFINIMPEKGV

\section{Secondary structure:}

\section{CEEEEECCCСССССНHНHННННННННСCCС- EEEEECCCCTTC}

A protein sequence affects the structure and function; in other words, a protein sequence determines its structure, and the structure determines functions. If amino acids in a protein sequence are arranged in a different order in the skeleton branch of the side chain $\mathrm{R}$ group, the nature of the protein would reveal specific functions. Even for different species of proteins, if they have a similar structure, their functions would be also similar. Therefore, predicting the protein structure is crucial to the function analysis. Besides, the secondary structure refers to the relative position of the space between the atoms of a certain backbone. Traditional protein structure determination was done by protein Xray crystallography or nuclear magnetic resonance (NMR). However, all experimental analysis costs much time. In order to shorten the time to help biologists, protein structure prediction by computers facilitates reaching this goal.

The prediction of protein secondary structure has been studied for decades. Early, the statistical analysis of secondary structure was done for a single amino acid. The most 
representative is the Chou and Fasman method [1], and the accuracy is only 50\%. Next, the statistical analysis for amino acid segments was done further. A segment length is usually with 9 21 amino acids. Based on an amino acid segment, predicting the structure of central residues enables promoting the accuracy. The most representative is the GOR method [2], and the accuracy increases more than $10 \%$ (about 63\%). At present, the prediction methods on protein secondary structure have evolved into using the PSI-BLAST program [3] to find the protein homology information, based on PSSM (position-specific scoring matrices) profiles. The accuracy of using PSSM to predict secondary structure has reached between 70 and $80 \%$ [4-7]. However, we believe that there still exists a great improvement in predicting protein secondary structure.

The rest of this paper is organized as follows. In Section 2, basic concepts used in the proposed methods are introduced first. In Section 3, we propose the methods and relevant features to predict the secondary structure of a protein sequence. Then, we make use of window sizes and tune parameters in the experiments in Section 4, in order to obtain better experimental results. Finally, we make a conclusion in Section 5 .

\section{Basic Concepts}

2.1. Protein Secondary Structure. Protein secondary structure derived from the experimentally determined 3D structure has been defined using DSSP (Dictionary of Secondary Structures of Proteins) [8], STRIDE (STRuctural IDEntification) [9], and DEFINE (DEFINE_structure) [10]. DSSP is selected here so that our method can be compared with most existing methods, based on the same protein secondary structure definition. Eight secondary structure classes were defined there, that is, $\mathrm{H}(\alpha$-helix $), \mathrm{G}(310$-helix $), \mathrm{I}(\pi$-helix $)$, $\mathrm{E}(\beta$-strand), $\mathrm{B}$ (isolated $\beta$-bridge), $\mathrm{T}($ turn $), \mathrm{S}($ bend $)$, and -(rest). The eight structure classes are usually reduced to three classes of helix $(\mathrm{H})$, sheet $(\mathrm{E})$, and coil (C). Five reductions could be performed as follows:

(1) H, G and I to H; E to E; the rest to C

(2) H, G to H; E, B to E; the rest to C

(3) $\mathrm{H}, \mathrm{G}$ to $\mathrm{H}$; E to $\mathrm{E}$; the rest to $\mathrm{C}$

(4) $\mathrm{H}$ to $\mathrm{H} ; \mathrm{E}, \mathrm{B}$ to $\mathrm{E}$; the rest to $\mathrm{C}$

(5) $\mathrm{H}$ to $\mathrm{H}$; E to $\mathrm{E}$; the rest to $\mathrm{C}$.

The first reduction was used in the PHD (Profile network from HeiDelberg) method [7] which is the early secondary structure prediction method using multiple sequence alignments of proteins homologous with a query protein sequence. We also use the first reduction in order to provide a fair comparison with other prediction methods.

2.2. SVM (Support Vector Machine). SVM was first investigated by Boser et al. in 1992 [11]. It solves linearly inseparable problems by nonlinearly mapping the vector in a low dimensional space to a higher dimensional feature space and constructs an optimal hyper-plane in the higher dimensional space. Therefore, SVM has high performances in data classification. A classification task usually involves with training and testing data which consist of some data instances. Each instance in the training set contains one "target value" (i.e., class label) and several "attributes" (i.e., features). The goal of SVM is to produce a model which can predict the target value of data instances in the testing set by using the attributes.

\section{Methods and Features}

3.1. System Architecture. The system architecture of predicting protein secondary structure is divided into three steps, as illustrated in Figure 1. The first step is to determine/extract the relevant features in/from protein sequences. Then, in the second step, we feed the features into SVM, respectively, in the training and test phases. Finally, we use a filter method to refine the predicted results from the trained SVM. During the SVM training phase, we not only train the SVM using the training data, but also, in advance, find the optimal sliding window size and the cost and gamma parameters of SVM kernel function, using the entire data set. The details about each module in the system architecture are depicted in the following subsections.

3.2. Feature Extraction. Five relevant kinds of features are extracted from protein sequences to predict protein secondary structure, that is, (1) conformation parameters, (2) position specific scoring matrix (PSSM) profiles, (3) net charge, (4) hydrophobic, and (5) side chain mass. The process of feature extraction is shown in Figure 2.

3.2.1. Extracting Sequences. First, we extract amino acid and secondary structure sequences from the PDB website (http://www.rcsb.org/pdb/home/home.do), using the PDB codes of CB513 [12]. Then, we can further extract five different features from amino acid sequences as follows.

3.2.2. Conformation Parameters. Conformation parameters are the proportions that residues (or amino acids) tend to secondary structure. In general, protein secondary structure is divided into three types: $\alpha$-helix $(\mathrm{H}), \beta$-sheet $(\mathrm{E})$, and coil (C), so that there are three values for each amino acid. In the feature extraction, all the conformation parameters are calculated from a data set. The conformation parameters for each amino acid $S_{i j}$ are defined as follows:

$$
S_{i j}=\frac{a_{i j}}{a_{i}}, \quad \text { where } i=1, \ldots, 20, j=1,2,3 .
$$

In this formula, $i$ indicates the 20 amino acids, and $j$ indicates the 3 types of secondary structure: $\mathrm{H}, \mathrm{E}$, and C. Here, $a_{i}$ is the amount of the $i$ th amino acid in a data set whereas $a_{i j}$ is the amount of the $i$ th amino acids with the $j$ th secondary structure. The conformation parameters for each amino acid in a data set are shown in Table 1 . The reason of using conformation parameters as features is that the folding of each residue has some correlation with forming a specific structure. 


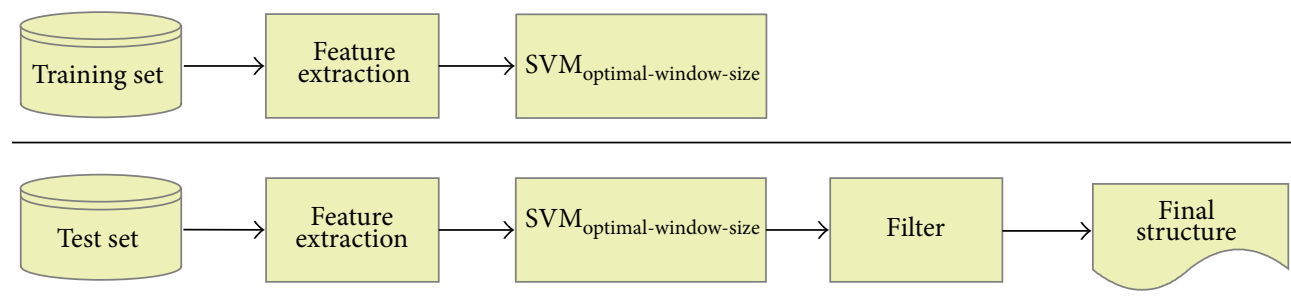

FIGURE 1: System architecture.

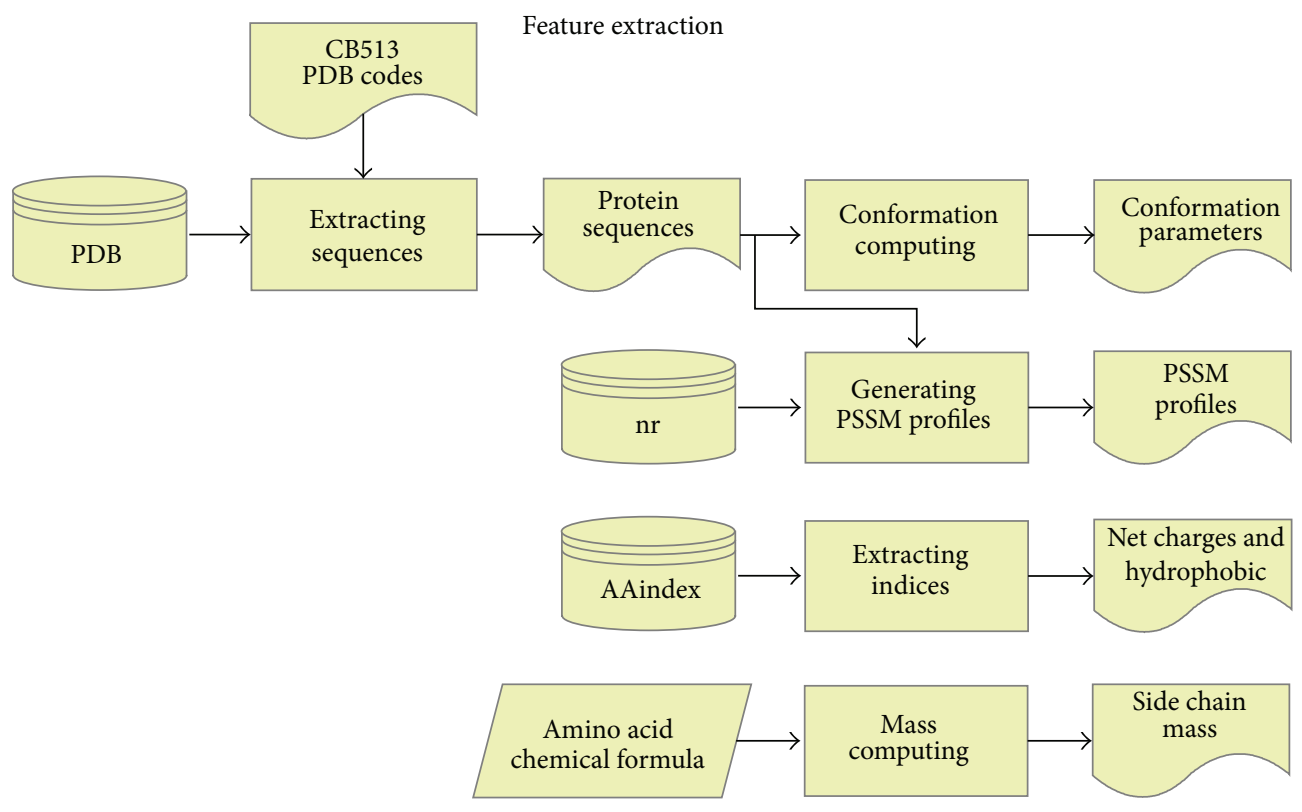

Figure 2: Process of feature extraction.

3.2.3. PSSM Profiles. PSSM profiles are generated by PSIBLAST (Position Specific Iterative-Basic Local Alignment Search Tool) program. Since PSSM profiles are involved with biological evolution, we consider them as features in our work. A PSSM profile has $L \times 20$ elements, where $L$ is the length of a query sequence. These profiles are then used as the input features to feed an SVM, employing a sliding window method.

PSI-BLAST is based on BLAST which has been published by Altschul et al. in 1997 [3]. Since PSI-BLAST program is more sensitive than other methods, we can find a lot of low similarity sequences and similarity structure function of protein sequences. First, a database containing all known sequences (or nonredundant database) is selected. Then, low complexity regions are removed from the nr database. Finally, PSI-BLAST program is used to query each sequence in CB513 and generates PSSM profiles after three iterations. Here, multiple sequence alignment (MSA) and BLOSUM62 matrix [13] are used in this process.

The reason of using the sliding window method is to get more surrounding information of residues. We consider a sliding window of size 7 19 at which a predicted residue is centered to extract input features. The optimal window size yielding favorable predictive performances would be obtained experimentally. For the $i$ th residue centered at the sliding window of size 7 , we can get $7 \times 20$ features $F_{i+n, j}$ where $n$ is in the range $[-3,3]$ and $j$ is the PSSM column from 1 to 20 .

3.2.4. Net Charges. There are five amino acids with charges, that is, R, D, E, H, and K. Since residues with similar electric charges repel each other and interrupt the hydrogen bond of main chain, they are adverse to $\alpha$-helix formation. Besides, the continuous residues of $\beta$-sheet cannot be with similar charges. This information facilitates predicting the secondary structure. The net charge of amino acids can be taken from Amino Acid index database (or AAindex) [14-18], as shown in Table 2. A plus sign represents a positive charge and a minus sign represents a negative charge.

3.2.5. Hydrophobic. For protein folding, polar residues prefer to stay outside of protein to prevent non-polar (hydrophobic) residues from exposing to polar solvent, like water. Therefore, hydrophobic residues appearing periodically can be used for predicting protein secondary structure. In general, the residues in $\alpha$-helix structure are made up of one segment of hydrophobic and one segment of hydrophilic. However, $\beta$ sheet structure is usually influenced by the environment, so 
TABLE 1: Conformation parameters for each amino acid in a data set.

\begin{tabular}{lccc}
\hline Amino acids & H & E & C \\
\hline A & 0.49 & 0.16 & 0.35 \\
R & 0.42 & 0.19 & 0.39 \\
N & 0.27 & 0.13 & 0.6 \\
D & 0.31 & 0.11 & 0.58 \\
C & 0.26 & 0.29 & 0.45 \\
E & 0.49 & 0.15 & 0.36 \\
Q & 0.46 & 0.16 & 0.38 \\
G & 0.16 & 0.14 & 0.7 \\
H & 0.3 & 0.22 & 0.48 \\
I & 0.35 & 0.37 & 0.28 \\
L & 0.45 & 0.24 & 0.31 \\
K & 0.4 & 0.17 & 0.43 \\
M & 0.44 & 0.23 & 0.33 \\
F & 0.35 & 0.3 & 0.35 \\
P & 0.18 & 0.09 & 0.74 \\
S & 0.28 & 0.19 & 0.54 \\
T & 0.25 & 0.27 & 0.48 \\
W & 0.37 & 0.29 & 0.35 \\
Y & 0.34 & 0.3 & 0.36 \\
V & 0.3 & 0.41 & 0.29 \\
\hline
\end{tabular}

TABLE 2: Net charge of amino acids.

\begin{tabular}{lc}
\hline Amino acids & Mass \\
\hline A & 0 \\
R & +1 \\
N & 0 \\
D & -1 \\
C & 0 \\
E & -1 \\
Q & 0 \\
G & 0 \\
H & 0 \\
I & +1 \\
L & 0 \\
K & 0 \\
M & 0 \\
F & +1 \\
P & 0 \\
S & 0 \\
T & 0 \\
W & 0 \\
Y & V \\
\hline
\end{tabular}

this phenomenon is not obvious. In other words, hydrophobic affects the stability of secondary structure. The hydrophobic values of amino acids can also be obtained from Amino Acid index database (or AAindex) [14-18], as shown in Table 3. The more positive values are, the more hydrophobic is.
TABLE 3: Hydrophobic values of amino acids.

\begin{tabular}{lc}
\hline Amino acids & Mass \\
\hline A & 1.8 \\
R & -4.5 \\
N & -3.5 \\
D & -3.5 \\
C & 2.5 \\
E & -3.5 \\
Q & -3.5 \\
G & -0.4 \\
H & -3.2 \\
I & 4.5 \\
L & 3.8 \\
K & -3.9 \\
M & 1.9 \\
F & 2.8 \\
P & -1.6 \\
S & -0.8 \\
T & -0.7 \\
W & -0.9 \\
Y & -1.3 \\
V & 4.2 \\
\hline
\end{tabular}

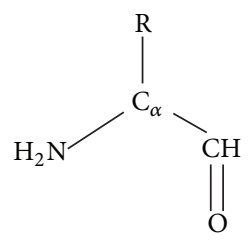

Figure 3: Basic structure of amino acids.

3.2.6. Side Chain Mass. Although the basic structure as shown in Figure 3 is the same for 20 amino acids, the size of the side chain $\mathrm{R}$ group still influences structure folding. Here, we explain the influences as follows. First, the side chain $\mathrm{R}$ group is distributed in the outside of the main chain of $\alpha$-helix structure, but the continuous large R groups can make $\alpha$-helix structure unstable, thereby disabling amino acids from forming $\alpha$-helix structure. Next, the $\mathrm{R}$ group with ring structure like proline $(\mathrm{P})$ is not easy to form $\alpha$-helix structure. Proline is composed of 5 atoms in a ring, which is not easy to reverse and is also not easy to generate a hydrogen bond. Finally, we observe that the $\mathrm{R}$ group of $\beta$-sheet structure is smaller than those of other structures, in general. Therefore, we include the side chain mass as a feature, as shown in Table 4.

3.3. SVM (Finding the Optimal Window Size and Parameters). The SVM used in the experiments is a classifier for predicting the secondary structure $\mathrm{H}, \mathrm{E}$, and $\mathrm{C}$. Threefold cross-validation is employed on the CB513 data set to find (1) the optimal window size in the range $[7,19]$ and (2) the optimal parameters of the kernel function, such as cost C and gamma $\gamma$. Here, the kernel function used in the SVM 
TABLE 4: Side chain mass of amino acids.

\begin{tabular}{lc}
\hline Amino acids & Mass \\
\hline A & 15.0347 \\
R & 100.1431 \\
N & 58.0597 \\
D & 59.0445 \\
C & 47.0947 \\
E & 73.0713 \\
Q & 72.0865 \\
G & 1.0079 \\
H & 81.0969 \\
I & 57.1151 \\
L & 57.1151 \\
K & 72.1297 \\
M & 75.1483 \\
F & 91.1323 \\
P & 41.0725 \\
S & 31.0341 \\
T & 45.0609 \\
W & 130.1689 \\
Y & 107.1317 \\
V & 43.0883 \\
\hline
\end{tabular}

is RBF (i.e., Radial Basis Function). To solve the multiclass problem confronted in the work, we employ the "one-againstone" approach. For 3 classes, we need 3 binary classifiers and set the labels of the secondary structure $(H, E, C)$ to $(-1,+1$, $+2)$. Then, we use the max-wins voting strategy to determine the class; in other words, each binary classifier casts a vote, and the winning class is with the highest number of votes. In the experiments, the LIBSVM tool kit proposed by Chang and Lin [19] would be used to implement the program. After the optimal window size and parameters are found, we would use the SVM for training and test.

3.4. Filter. A single residue in its natural state cannot be alone folded into $\alpha$-helix or $\beta$-sheet. Thus, setting thresholds on the length of consensus secondary structure can be used to filter out incorrect predicted results. For example, at least three contiguous residues are for $\alpha$-helix and at least two contiguous residues are for $\beta$-sheet. For the current scanning window $(i-1, i, i+1)$ in the predicted secondary structure, two possible structures could happen at position $i$ :

Case $H$ : if $\operatorname{str}(i-1)$ and $\operatorname{str}(i+1)$ are $H$, then $\operatorname{str}(i)$ is not changed; otherwise, extend the examined segment to $(i-3, i-2, i-1, i, i+1, i+2, i+3)$ and replace $\operatorname{str}(i)$ with the majority structure in the examined segment.

Case $\mathrm{E}$ : if $\operatorname{str}(i-1)$ or $\operatorname{str}(i+1)$ is $\mathrm{E}$, then $\operatorname{str}(i)$ is not changed; otherwise, extend the examined segment to $(i-3, i-2$, $i-1, i, i+1, i+2, i+3)$ and replace $\operatorname{str}(i)$ with the majority structure in the examined segment.

For the example as shown in Figure 4, after the filtering, $Q_{3}$ for 9 INSb is improved from 76.7 to 80 and SOV99 is

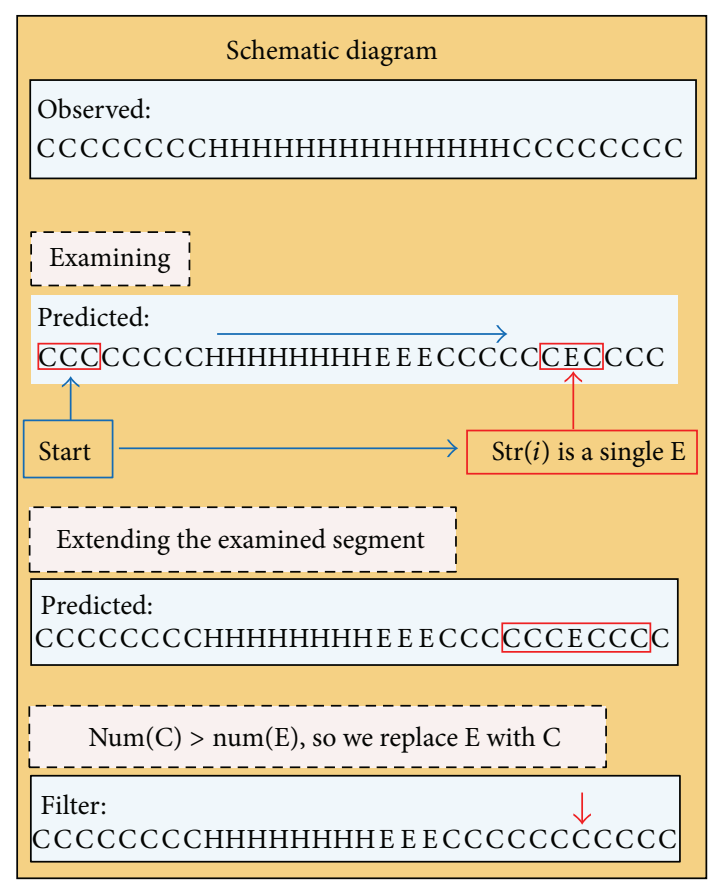

FIGURE 4: Schematic diagram for filtering 9INSb.

TABLE 5: Structures of the CB513 data set.

\begin{tabular}{lcccc}
\hline Structures & H & E & C & Total \\
\hline Residues & 29090 & 17950 & 37053 & 84093 \\
\hline
\end{tabular}

improved from 77.8 to 93.3 where $Q_{3}$ and SOV99 will be described in Section 4.2.

\section{Experiments}

4.1. Data Set. In the previous work, some typical data sets were frequently used in protein secondary structure prediction, such as RS126 [7], CB513 [12], CASP [20], and EVA [21]. Here, we consider the selected data set should be with low similarity; that is, the protein sequences within the data set are not similar to each other. Thus, the protein secondary structure prediction we develop would enable predicting an unknown protein sequence more accurately.

In our work, the data set we choose is nonhomologous CB513 data set constructed by Cuff and Barton and contains 513 protein chains. Almost all the sequences in the RS126 data set are also included in the CB513 data set. The CB513 data set contains 16 chains of $\leqq 30$ residues. Although very short chains would slightly decrease the accuracy for the hard definition of secondary structures, we still include them in the set for comprehensive study. We retrieve the CB513 data set from the website: http://paraschopra.com/projects/evoca_prot/index.php, which contains 84,093 residues where $34.59 \%$ of the residues is for helix, $21.35 \%$ for sheet, and $44.06 \%$ for coil, as shown in Table 5.

4.2. Performance Measures. Two kinds of performance measures are frequently used in protein secondary structure 
prediction; that is, $Q_{3}$ or accuracy (three-state overall perresidue accuracy) and SOV99 [22] (or SOV94 [23]) (Segment Overlap measure). $Q_{3}$ is a residue-based measure of threestructure overall percentage of correctly classified residues, which can be represented as

$$
Q_{3}=\frac{\mathrm{H}_{\text {pre }}+\mathrm{E}_{\text {pre }}+\mathrm{C}_{\text {pre }}}{N_{\text {total }}},
$$

where $N_{\text {total }}$ is the total number of predicted residues, $\mathrm{H}_{\text {pre }}$ is the correctly classified secondary structure for helix, $\mathrm{E}_{\text {pre }}$ for sheet, and $\mathrm{C}_{\text {pre }}$ for coil.

SOV99 is a segment-based measure of three structures, whose value is within the range $[0,100]$, as shown in Formula (3). SOV99 differs from $Q_{3}$ in the prediction unit such that SOV99 would penalize wrong predictions; for example, a single helix predicted as a multiply-split helix is unrealistic prediction

$$
\begin{aligned}
\mathrm{SOV}=100 \times\left[\frac{1}{N} \sum_{i \in\{\mathrm{H}, \mathrm{E}, \mathrm{C}\}} \sum_{S(i)} \frac{\min o v\left(s_{1}, s_{2}\right)+\delta\left(s_{1}, s_{2}\right)}{\max o v\left(s_{1}, s_{2}\right)}\right. \\
\left.\times \operatorname{len}\left(s_{1}\right)\right],
\end{aligned}
$$

where $s_{1}$ and $s_{2}$ denote segments of secondary structure $i$ $(\mathrm{H}, \mathrm{E}$, or $\mathrm{C}), S(i)=\left\{\left(s_{1}, s_{2}\right): s_{1} \cap s_{2} \neq \varnothing, s_{1}\right.$ and $s_{2}$ are both in structure $i$ \}, $N$ is a normalization value, $\min o v\left(s_{1}, s_{2}\right)$ is the length of actual overlap of $s_{1}$ and $s_{2}, \max o v\left(s_{1}, s_{2}\right)$ is the length of total extent for $s_{1}$ and $s_{2}$, and $\delta\left(s_{1}, s_{2}\right)$ can be represented as

$$
\delta\left(s_{1}, s_{2}\right)=\min \left\{\begin{array}{c}
\max o v\left(s_{1}, s_{2}\right)-\min o v\left(s_{1}, s_{2}\right) \\
\min \text { ov }\left(s_{1}, s_{2}\right) \\
\operatorname{int}\left(\frac{\operatorname{len}\left(s_{1}\right)}{2}\right) \\
\operatorname{int}\left(\frac{\operatorname{len}\left(s_{2}\right)}{2}\right) .
\end{array}\right\} .
$$

The definition of $\delta$ and the normalization value $\mathrm{N}$ are different for SOV99 and SOV 94.

4.3. Optimal Parameters and Window Sizes. As introduced in Section 2.2, we adopt the well-known LIBSVM developed by Chang and Lin [19] as an SVM classifier. The kernel function used here is RBF (Radial Basis Function) since it is more accurate and effective than the other kernel ones. The parameters $\mathrm{C}$ and $\gamma$ are determined by the optimum performance of $6 \times 6$ combinations between $\left[2^{0}, \ldots, 2^{5}\right]$ and $\left[2^{-6}, \ldots, 2^{-1}\right]$ for each window size. Moreover, the feature vector is normalized in the range $[0,1]$ and the number of features in a larger window size would become more. The optimal parameters and classification accuracy are evaluated in threefold cross-validation, as shown in Table 6.

According to the experimental results, we found the optimal parameters and window size are $C=2^{1}, \gamma=2^{-4}$, and $\mathrm{WS}=13$. Then, we use these parameters and window size to conduct the further experiments.
TABLE 6: Optimal parameters for different window sizes.

\begin{tabular}{lcccc}
\hline Window sizes & Features & Best $C$ & Best $\gamma$ & Accuracy (\%) \\
\hline 7 & 146 & $2^{0}$ & $2^{-3}$ & 76.3203 \\
9 & 186 & $2^{1}$ & $2^{-4}$ & 76.7935 \\
11 & 226 & $2^{0}$ & $2^{-4}$ & 77.4464 \\
13 & 266 & $2^{1}$ & $2^{-4}$ & 78.0029 \\
15 & 306 & $2^{1}$ & $2^{-4}$ & 77.7806 \\
17 & 346 & $2^{1}$ & $2^{-5}$ & 77.6549 \\
19 & 386 & $2^{1}$ & $2^{-4}$ & 77.5796 \\
\hline
\end{tabular}

TABLE 7: Confusion matrix without filtering.

\begin{tabular}{lcccc}
\hline \multirow{2}{*}{ Actual } & \multicolumn{4}{c}{ Predicted } \\
& $\mathrm{H}$ & $\mathrm{E}$ & $\mathrm{C}$ & Recall (\%) \\
\hline $\mathrm{H}$ & 22976 & 931 & 5183 & 78.98 \\
$\mathrm{E}$ & 1044 & 11569 & 5337 & 64.45 \\
$\mathrm{C}$ & 3451 & 3059 & 30543 & 82.43 \\
Precision (\%) & 83.64 & 74.36 & 74.38 & 77.40 \\
\hline
\end{tabular}

TABLE 8: Confusion matrix with filtering.

\begin{tabular}{lcccc}
\hline \multirow{2}{*}{ Actual } & \multicolumn{4}{c}{ Predicted } \\
& $\mathrm{H}$ & $\mathrm{E}$ & $\mathrm{C}$ & Recall (\%) \\
\hline $\mathrm{H}$ & 22372 & 818 & 5900 & 76.91 \\
$\mathrm{E}$ & 432 & 11776 & 5742 & 65.60 \\
$\mathrm{C}$ & 1514 & 2819 & 32720 & 88.31 \\
Precision (\%) & 92.00 & 76.40 & 73.76 & 79.52 \\
\hline
\end{tabular}

4.4. Experimental Results. In this section, we compare the experimental results without filtering and with filtering. For the classification results, a confusion matrix is employed to present the correct and false predictions based on the precision and recall, as shown in Tables 7 and 8 . The precision and recall are expressed as follows:

Precision $(i)$

$$
\begin{aligned}
& =\frac{\text { The number of correctly classified structure } i}{\text { The number of total predicted structure } i}, \\
& \qquad \text { for } i=\mathrm{H}, \mathrm{E}, \mathrm{C},
\end{aligned}
$$

$\operatorname{Recall}(i)$

$$
=\frac{\text { The number of correctly classified structure } i}{\text { The number of total actual structure } i},
$$

$$
\text { for } i=\mathrm{H}, \mathrm{E}, \mathrm{C} \text {. }
$$

Obviously, the classification accuracy with filtering (i.e., $79.52 \%$ ) is higher than that without filtering (i.e., 77.40\%). The precision for $\mathrm{H}$ and the recall for $\mathrm{C}$ especially are improved from 83.64 to 92.00 (with filtering) and from 82.43 to 88.31 (with filtering), respectively. Therefore, the filter rules are required to improve the accuracy in predicting protein secondary structure. 
TABLE 9: Comparisons between ours and other methods.

\begin{tabular}{|c|c|c|c|c|c|c|}
\hline Methods & $Q_{3}$ & SOV94 & SOV99 & $\mathrm{R}(\mathrm{H})$ & $\mathrm{R}(\mathrm{E})$ & $\mathrm{R}(\mathrm{C})$ \\
\hline PHD (RS126) [7] & 70.8 & 73.5 & - & 72.0 & 66.0 & 72.0 \\
\hline SVMfreq (RS126) [5] & 71.2 & 74.6 & - & 73.0 & 58.0 & 73.0 \\
\hline SVMfreq (CB513) [5] & 73.5 & 76.2 & - & 75.0 & 60.0 & 79.0 \\
\hline PMSVM (CB513) [4] & 75.2 & 80.0 & - & 80.4 & 71.5 & 72.8 \\
\hline SVMpsi (RS126) [6] & 76.1 & 79.6 & 72.0 & 77.2 & 63.9 & 81.5 \\
\hline SVMpsi (CB513) [6] & 76.6 & 80.1 & 73.5 & 78.1 & 65.6 & 81.1 \\
\hline Ours without filtering (CB513) & 77.40 & 90.20 & 71.10 & 78.98 & 64.45 & 82.43 \\
\hline Ours with filtering (CB513) & 79.52 & 86.10 & 74.60 & 76.91 & 65.60 & 88.31 \\
\hline
\end{tabular}

4.5. Comparing with Other Methods. Here, we compare our methods with other four methods; that is, PHD, SVMfreq, PMSVM, and SVMpsi as shown in Table 9. Both the PHD and SVMfreq methods are based on the frequency profiles with multiple sequence alignment; however, the classifier used in the PHD method is a neural network (or NN) whereas the classifier used in the SVMfreq method is a support vector machine (or SVM). Similarly, both the PMSVM and SVMpsi methods are based on the PSSM profiles generated from PSIBLAST. Although they use the same-type classifier (or SVM), the former adopts one-versus-one classifier (i.e., H/E, E/C, $\mathrm{C} / \mathrm{H}$ ) and the latter adopts the one-versus-rest classifier (i.e., $\mathrm{H} / \sim \mathrm{H}, \mathrm{E} / \sim \mathrm{E}, \mathrm{C} / \sim \mathrm{C})$.

As shown in Table 9, we found that all the performance measures of our method (i.e., the version with filtering), including $Q_{3}$, SOV94, and SOV99, are higher than those of the other four methods, regardless using the CB513 or RS126 data sets. $Q_{3}$ for the version with filtering (or without filtering) is improved by 2.92 (or 0.8 ), SOV94 for the version with filtering (or without filtering) is improved by 6 (or 10.1), and SOV99 for the version with filtering is improved by 1.1, compared with the results of the SVMpsi method for CB513 (i.e., the next best one).

However, our method (i.e., the version with filtering) has lower $\mathrm{R}(\mathrm{H})$ than the SVMpsi method (i.e., 76.91 versus 78.1). One of the possible reasons is that the threshold on the length of consensus secondary structure (i.e., at least three contiguous residues for $\mathrm{H}$ ) is set in the filter. Although the recall for $\mathrm{H}$ is decreased, the predicted structures are more structurally meaningful. Besides, we found that two SOV measures in the SVMpsi and our methods vary greatly. Although SOV94 is decreased (i.e., from 90.20 to 86.10 ) after applying the filter in our method, the latest definition (i.e., SOV99) is still the highest.

\section{Conclusions}

In this paper, we propose a protein secondary structure prediction method using PSSM profiles and four physicochemical features, including conformation parameters, net charges, hydrophobic, and side chain mass. In the experiments, the SVM with the optimal window size and the optimal parameters of the kernel function is found first. Then, we train the SVM using the PSSM profiles and physicochemical features extracted from the CB513 data set.
Finally, we use the filter to refine the predicted results from the trained SVM. For the experimental results, $Q_{3}$, SOV94, SOV99, and recall of our method are higher than those of the SVMpsi method based on the PSI-BLAST profiles as well as the SVMfreq method based on the frequency profiles with multiple sequence alignment for the CB513 data set. In summary, considering these physicochemical features in predicting protein secondary structure would exhibit better performances.

\section{Acknowledgment}

This work was supported by National Science Council of Taiwan under Grant NSC100-2218-E-224-011-MY3.

\section{References}

[1] P. Y. Chou and G. D. Fasman, "Empirical predictions of protein conformation," Annual Review of Biochemistry, vol. 47, pp. 251276, 1978.

[2] J. Garnier, D. J. Osguthorpe, and B. Robson, "Analysis of the accuracy and implications of simple methods for predicting the secondary structure of globular proteins," Journal of Molecular Biology, vol. 120, no. 1, pp. 97-120, 1978.

[3] S. F. Altschul, T. L. Madden, A. A. Schäffer et al., "Gapped BLAST and PSI-BLAST: a new generation of protein database search programs," Nucleic Acids Research, vol. 25, no. 17, pp. 3389-3402, 1997.

[4] J. Guo, H. Chen, Z. Sun, and Y. Lin, "A novel method for protein secondary structure prediction using dual-layer SVM and profiles," Proteins, vol. 54, no. 4, pp. 738-743, 2004.

[5] S. Hua and Z. Sun, "A novel method of protein secondary structure prediction with high segment overlap measure: support vector machine approach," Journal of Molecular Biology, vol. 308, no. 2, pp. 397-407, 2001.

[6] H. Kim and H. Park, "Protein secondary structure prediction based on an improved support vector machines approach," Protein Engineering, vol. 16, no. 8, pp. 553-560, 2003.

[7] B. Rost and C. Sander, "Prediction of protein secondary structure at better than 70\% accuracy," Journal of Molecular Biology, vol. 232, no. 2, pp. 584-599, 1993.

[8] W. Kabsch and C. Sander, "Dictionary of protein secondary structure: pattern recognition of hydrogen-bonded and geometrical features," Biopolymers, vol. 22, no. 12, pp. 2577-2637, 1983. 
[9] D. Frishman and P. Argos, "Knowledge-based protein secondary structure assignment," Proteins, vol. 23, no. 4, pp. 566579, 1995.

[10] F. M. Richards and C. E. Kundrot, "Identification of structural motifs from protein coordinate data: secondary structure and first-level supersecondary structure," Proteins, vol. 3, no. 2, pp. 71-84, 1988.

[11] B. E. Boser, I. M. Guyon, and V. N. Vapnik, "Training algorithm for optimal margin classifiers," in Proceedings of the 5th Annual ACM Workshop on Computational Learning Theory, pp. 144152, July 1992.

[12] J. A. Cuff and G. J. Barton, "Evaluation and improvement of multiple sequence methods for protein secondary structure prediction," Proteins, vol. 34, no. 4, pp. 508-519, 1999.

[13] S. Henikoff and J. G. Henikoff, "Amino acid substitution matrices from protein blocks," Proceedings of the National Academy of Sciences of the United States of America, vol. 89, no. 22, pp. 10915-10919, 1992.

[14] S. Kawashima, H. Ogata, and M. Kanehisa, "AAindex: amino acid index database," Nucleic Acids Research, vol. 27, no. 1, pp. 368-369, 1999.

[15] S. Kawashima and M. Kanehisa, "AAindex: amino acid index database," Nucleic Acids Research, vol. 28, no. 1, p. 374, 2000.

[16] S. Kawashima, P. Pokarowski, M. Pokarowska, A. Kolinski, T. Katayama, and M. Kanehisa, "AAindex: Amino acid index database, progress report 2008," Nucleic Acids Research, vol. 36, no. 1, pp. D202-D205, 2008.

[17] K. Nakai, A. Kidera, and M. Kanehisa, "Cluster analysis of amino acid indices for prediction of protein structure and function," Protein Engineering, Design and Selection, vol. 2, no. 2, pp. 93-100, 1988.

[18] K. Tomii and M. Kanehisa, "Analysis of amino acid indices and mutation matrices for sequence comparison and structure prediction of proteins," Protein Engineering, vol. 9, no. 1, pp. 2736, 1996.

[19] C.-C. Chang and C.-J. Lin, "LIBSVM: a library for support vector machines," http://www.csie.ntu.edu.tw/ cjlin/libsvm, 2001.

[20] J. Moult, J. T. Pedersen, R. Judson, and K. Fidelis, "A large-scale experiment to assess protein structure prediction methods," Proteins, vol. 23, no. 3, pp. ii-iv, 1995.

[21] V. A. Eyrich, M. A. Martí-Renom, D. Przybylski et al., "EVA: continuous automatic evaluation of protein structure prediction servers," Bioinformatics, vol. 17, no. 12, pp. 1242-1243, 2002.

[22] A. Zemla, C. Venclovas, K. Fidelis, and B. Rost, "A modified definition of SOV, a segment based measure for protein secondary structure prediction assessment," Proteins, vol. 34, no. 2, pp. 220-223, 1999.

[23] B. Rost, C. Sander, and R. Schneider, "Redefining the goals of protein secondary structure prediction," Journal of Molecular Biology, vol. 235, no. 1, pp. 13-26, 1994. 

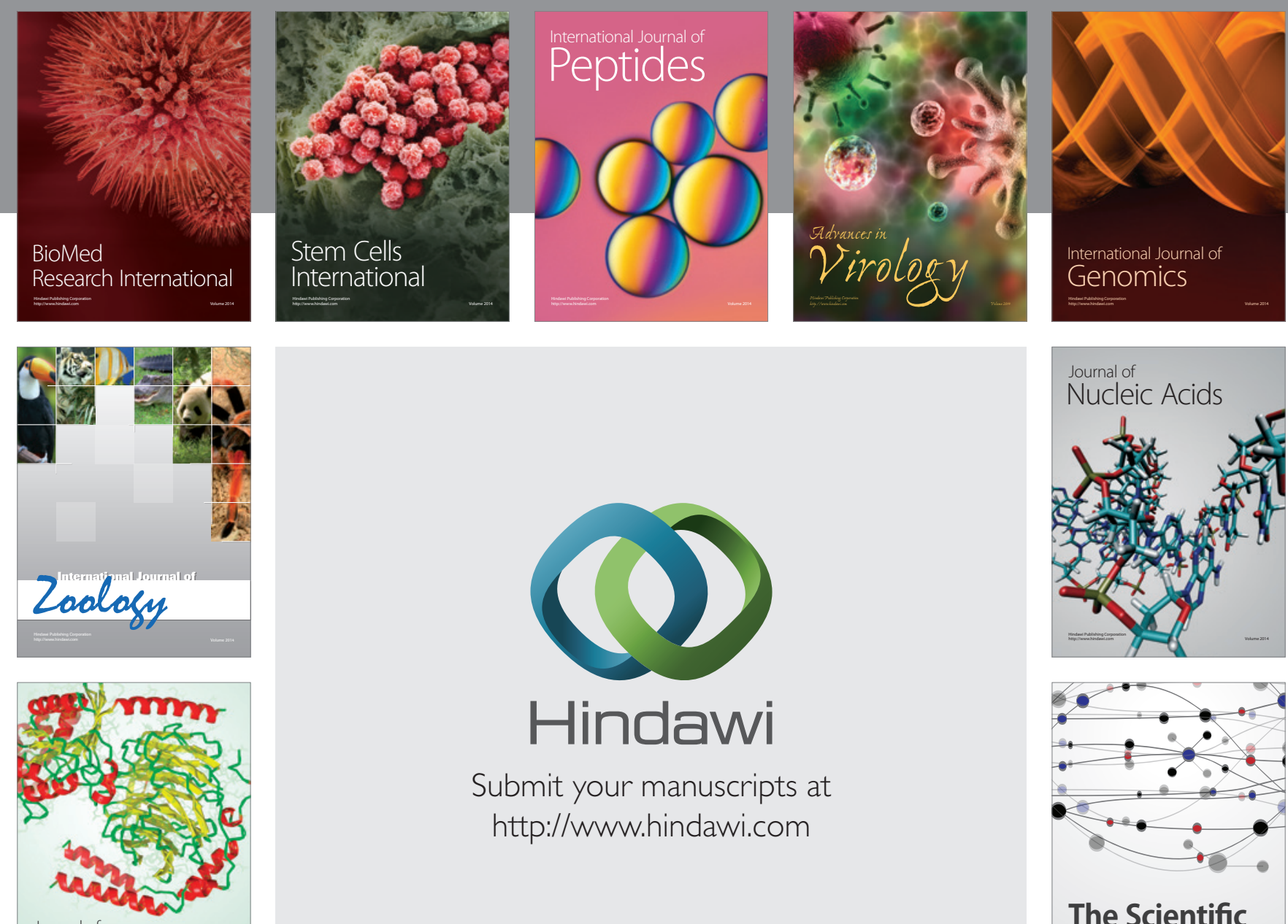

Submit your manuscripts at

http://www.hindawi.com

Journal of
Signal Transduction
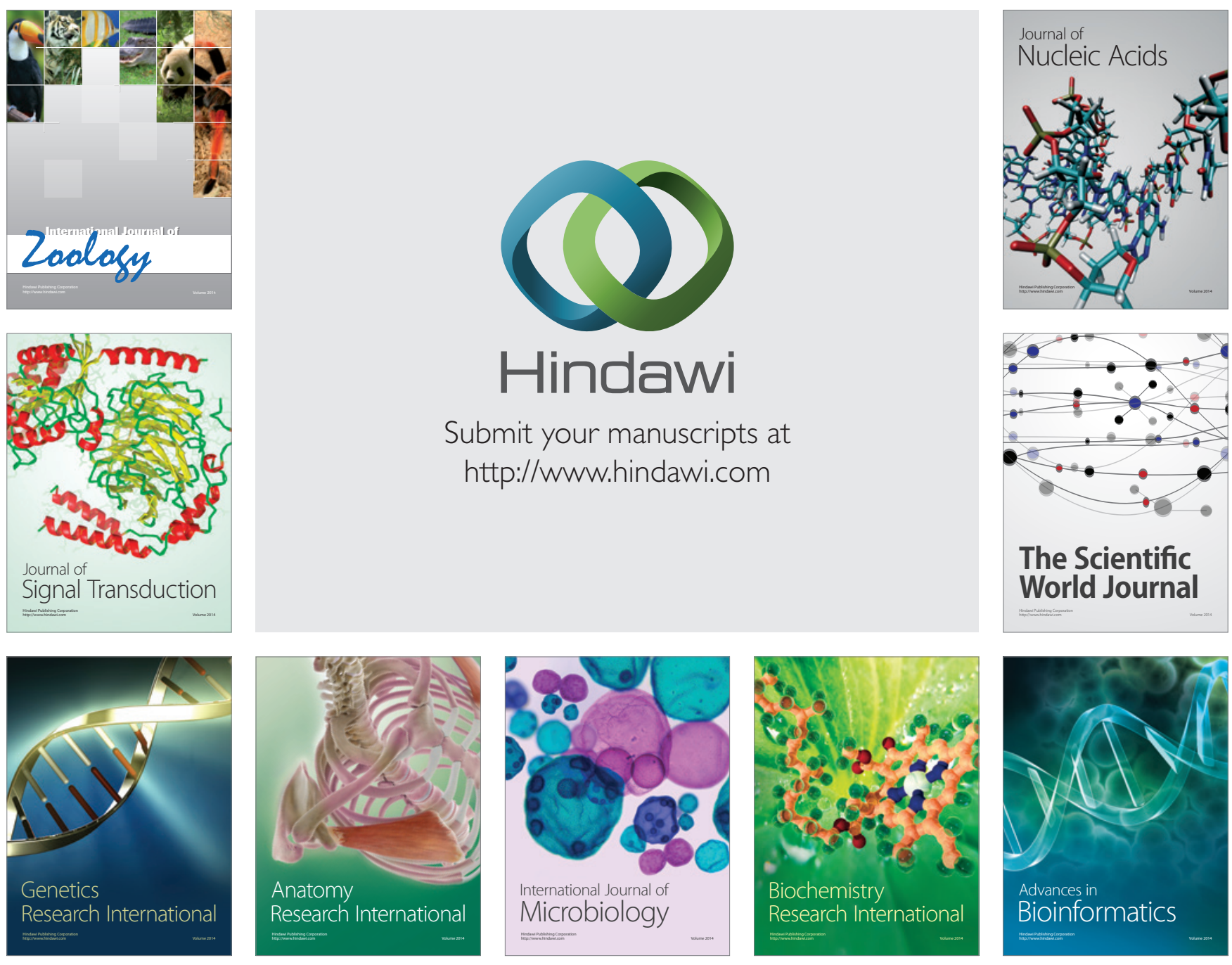

The Scientific World Journal
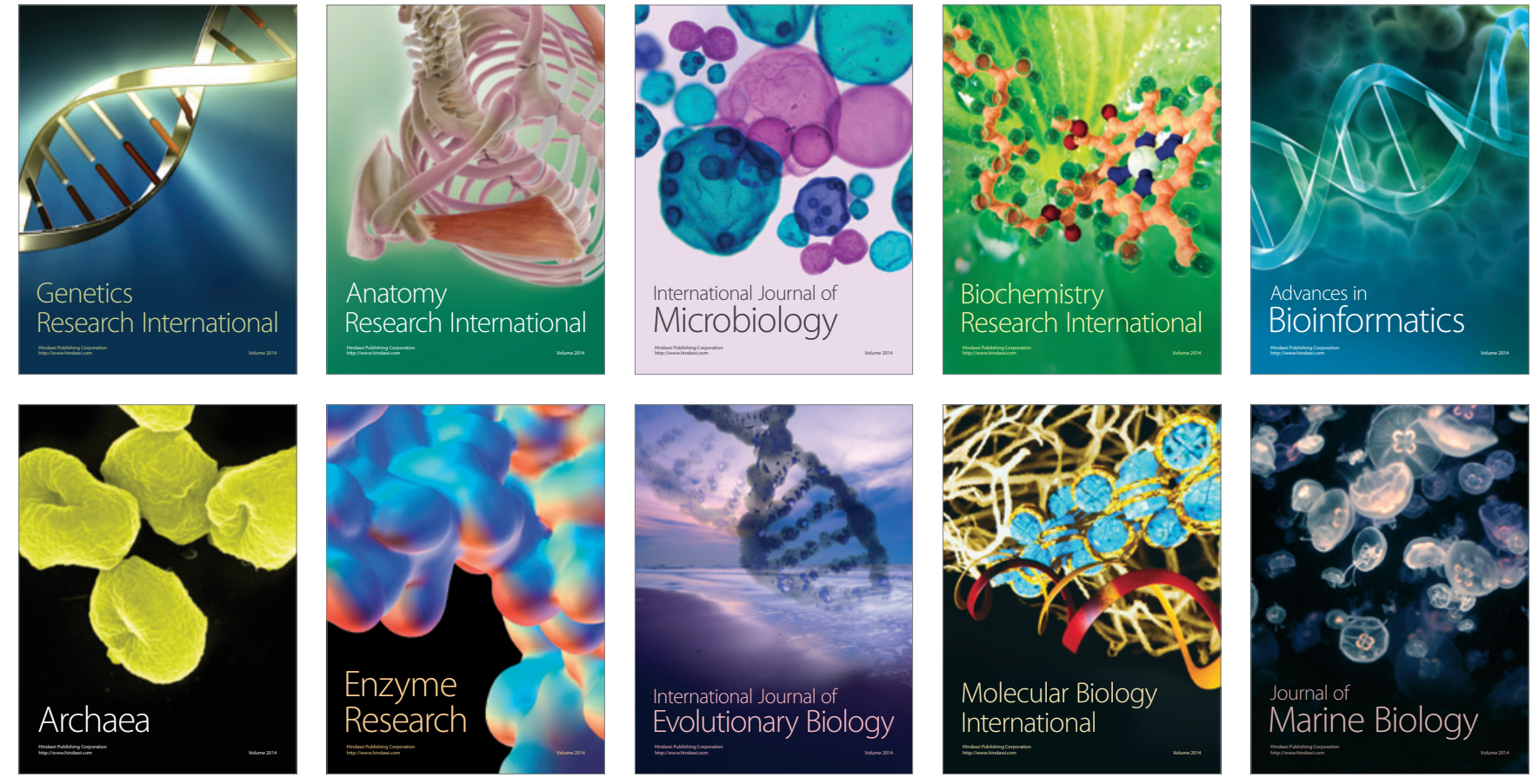